\title{
Erratum to: Physical Activity, Decision-Making Abilities, and Eating Disturbances in Pre- and Postbariatric Surgery Patients
}

\author{
Merle Bartsch $^{1}$ - Svenja Langenberg ${ }^{1} \cdot$ Kerstin Gruner-Labitzke $^{2} \cdot$ Mareike Schulze $^{3}$. \\ Hinrich Köhler $^{2}$ - Ross D. Crosby ${ }^{4}$. Michael Marschollek ${ }^{3}$. Martina de Zwaan ${ }^{1}$ • \\ Astrid Müller ${ }^{1}$
}

Published online: 14 September 2016

(C) Springer Science+Business Media New York 2016

\section{Erratum to: OBES SURG \\ DOI 10.1007/s11695-016-2208-y}

The published Abstract includes a mistake with regard to the percentages. The correct sentence is:

Methods The study included 71 bariatric surgery candidates (78 \% women, BMI $\left[\mathrm{kg} / \mathrm{m}^{2}\right] M=46.9, S D=6.0$ ) and 73 postoperative patients (78 \% women, BMI $M=32.0, S D=4.1$; $89 \%$ Roux-en-Y gastric bypass, $11 \%$ sleeve gastrectomy; months postoperative $M=8.2, S D=3.5$; total weight loss [\%] $M=33.2, S D=8.9$ ) who completed SenseWear $\mathrm{Pro}_{2}$ activity monitoring.

The online version of the original article can be found at http://dx.doi. org/10.1007/s11695-016-2208-y.

\section{Astrid Müller}

Mueller.Astrid@mh-hannover.de

1 Department of Psychosomatic Medicine and Psychotherapy, Hannover Medical School, Carl-Neuberg-Str. 1,

30625 Hannover, Germany

2 Department of Surgery, Herzogin Elisabeth Hospital, Braunschweig, Germany

3 Peter L. Reichertz Institut for Medical Informatics, University of Braunschweig - Institute of Technology and Hannover Medical School, Hannover, Germany

$4 \quad$ Neuropsychiatric Research Institute and Department of Psychiatry and Behavioral Science, University of North Dakota School of Medicine and Health Sciences, Fargo, ND, USA 